\title{
Archivos

\section{Valoración cardiovascular en niños y su relación con la altitud}

\section{Cardiovascular evaluation in children and its relation to altitude}

\author{
Julio Erdmenger Orellana*
}

\author{
Departamento de Cardiología, Hospital Infantil de México Federico Gómez, México DF, México
}

Recuerdo el caso de José, un chico de 8 años, atlético, con gran habilidad para jugar al futbol y el sueño personal de ser el siguiente «Chicharito», quien fue llevado por su padre al consultorio con el propósito de obtener respuesta a la siguiente pregunta:

¿Doctor mi hijo puede hacer ejercicio libremente y de manera segura o requiere de estudios especiales?

Esta pregunta es, indudablemente, un reto difícil de resolver para el cardiólogo pediatra. En mi opinión, la respuesta debe estar fundamentada en un número importante de variables como son entre otras: edad, antecedentes de enfermedad cardiaca o pulmonar, tipo de ejercicio, altitud donde se va a realizar, exploración física y estudios diagnósticos. La importancia de la respuesta es que de ella dependerá, en algunos casos, el desarrollo deportivo de chicos con un potencial especial; el reto es hasta dónde indicar estudios diagnósticos que permitan identificar condiciones de riesgo para ejercicio libre y de alto rendimiento en niños.

El trabajo publicado por Ilarraza-Lomelí et al. ${ }^{1}$ en $\mathrm{mi}$ opinión tiene importancia significativa para los cardiólogos pediatras, cardiólogos y médicos del deporte porque considera, sin ser el objetivo primordial del mismo, varios aspectos que lo fortalecen para ser un trabajo de referencia en el estudio de niños candidatos a realizar deporte. Entre esos varios aspectos están:

\section{a. Influencia de la altura sobre el nivel del mar}

Desde hace más de 70 años se iniciaron investigaciones sobre el efecto que tiene la altura sobre el nivel del mar en el rendimiento de deportistas; ahora se sabe que

\footnotetext{
* Dr. Márquez N. ${ }^{\circ}$ 162, Col. Doctores. Del. Cuauhtémoc. Teléfono: +52289917.

Correo electrónico: erdmenger@gmail.com
}

la presión y densidad atmosférica disminuyen de forma exponencial con la altitud, lo que conlleva una reducción en la presión parcial de $\mathrm{O}_{2}$ del aire y, asimismo, una disminución en la tensión de $\mathrm{O}_{2}$ en la sangre arterial. Es decir, la molécula de oxígeno ejerce menos presión para entrar en la sangre, por lo que se produce una falta de oxígeno (hipoxia) relativa. La presión barométrica disminuye en un $20 \%$ a 2,000 $\mathrm{m}$ y la presión parcial alveolar de oxígeno en un $18 \%$ a la misma altura ${ }^{2}$. El efecto que este factor produce en pruebas de más de 2 min realizadas en altitud media ha sido valorado en pacientes adultos ${ }^{3}$.

Terrados $^{4}$ señala una clasificación de la altitud dependiendo de criterios biológicos:

Baja altitud: hasta $1,000 \mathrm{~m}$ sobre el nivel del mar. En ella no se producen modificaciones fisiológicas en reposo, ni durante el ejercicio.

Media altitud: hasta 2,000 m; se producen ligeros efectos sobre todo durante la actividad deportiva.

Alta altitud: hasta 5,500 m, donde se producen efectos en reposo y durante la actividad deportiva.

Muy alta altitud: por encima de $5,500 \mathrm{~m}$, donde el efecto negativo es muy alto.

En medios deportivos se considera como altitud moderada la situada entre 1,500 y 3,000 m (alrededor de $2,000 \mathrm{~m}$ como altura ideal), objeto de la mayoría de los estudios sobre entrenamiento en altitud. Actualmente hay toda una ciencia que permite a los atletas realizar cambios en varios aspectos con el propósito de disminuir el efecto de la altura en su rendimiento.

Me parece que una fortaleza del estudio de IlarrazaLomelí et al. es el hecho de que los autores demuestran que la prueba de esfuerzo se puede aplicar de manera segura en niños a una altitud media sobre el nivel del mar y a edades tan temprana como los 4 años. 


\section{b. Prueba de esfuerzo en niños}

Desde hace varias décadas hay evidencia de los resultados diagnósticos y valor pronóstico de la prueba de esfuerzo en la evaluación cardiovascular del paciente adulto sano o enfermo; en niños, las publicaciones son escasas y en la literatura nacional prácticamente ausentes.

El artículo publicado también por llarraza-Lomelí ${ }^{5}$ en 2012 hace una excelente revisión de los aspectos teóricos de la prueba de ejercicio con análisis de gases espirados y su valor clínico; recomiendo su revisión para poder hacer un mejor análisis de los resultados presentados en el trabajo motivo de este comentario editorial.

En la literatura internacional hay trabajos que reportan resultados de prueba de esfuerzo en pacientes operados de cardiopatía congénita ${ }^{5,6}$. Indudablemente los resultados del trabajo de Ilarraza Lomelí et al. serán la base para la interpretación de pruebas de esfuerzo en grupos específicos de pacientes pediátricos mexicanos.

Siendo estos resultados de una aplicación clínica muy amplia en grupos específicos de pacientes, como son operados de cardiopatía congénita, con enfermedades respiratorias, hipertensión pulmonar primaria, enfermedad de Kawasaki, y dado que México ocupa el primer lugar mundial de obesidad infantil y se estima que cerca del $30 \%$ de los niños obesos tienen síndrome metabólico con el riesgo cardiovascular que esto implica, la población susceptible de aplicación de la prueba de esfuerzo en nuestro medio es muy alta, por lo que sería de gran utilidad que los autores incrementaran el número de sujetos de estudio por grupo etario para fortalecer los resultados del mismo.

\section{Bibliografía}

1. Ilarraza-Lomelí H, Castañeda-López J, Myers J, et al. Prueba de ejercicio cardiopulmonar en niños y adolescentes sanos, a una altitud moderadamente elevada. Arch Cardiol Mex. 2013;83:176-82.

2. Bichon M. Entrenamiento en altitud. Problemas, accidentes e incidentes. Cuadernos de atletismo. 1984;85:97-100.

3. Hollmann W. The historical development of altitude training and current medical knowledge. New studies in athletics. 1994;2:7-13.

4. Terrados N. El entrenamiento en altitud. INFOCOES. 1994;1:26-38.

5. Ilarraza-Lomelí H. Prueba de ejercicio con análisis de gases espirados. Arch Cardiol Mex. 2012;82:160-9.

6. Serra-Grima R, Doñate M, Borrás X, et al. Prueba de esfuerzo con función cardiopulmonar en niños operados de cardiopatía congénita. Recomendaciones de ejercicio físico en el ámbito escolar. Rev Esp Cardiol. 2011;64:780-7. 